\title{
Phase II study of temozolomide monotherapy in patients with extrapulmonary poorly differentiated neuroendocrine carcinoma that were resistant to platinum-based chemotherapy
}

Noritoshi Kobayashi ( $\nabla$ norikoba@yokohama-cu.ac.jp )

Yokohama City University Hospital https://orcid.org/0000-0002-9181-3722

Yuma Takeda

Yokohama City University Hospital

Naoki Okubo

Yokohama City University Hospital

Akihiro Suzuki

Yokohama City Unoversity Hospital

Motohiko Tokuhisa

Yokohama City University Hospital

Yukihiko Hiroshima

Yokohama City University Hospital

Yasushi Ichikawa

Yokohama City University Hospital

Research article

Keywords: Extraplumonary; poorly differentiated; neuroendocrine carcinoma; Temozolomide; phase II study;

Posted Date: June 22nd, 2020

DOI: https://doi.org/10.21203/rs.3.rs-31935/v1

License: (c) (1) This work is licensed under a Creative Commons Attribution 4.0 International License. Read Full License 


\section{Abstract}

Background: Extrapulmonary poorly differentiated neuroendocrine carcinoma (EPNEC) is a lethal disease with a poor prognosis. Platinum-based chemotherapy is used as the standard first-line treatment for unresectable EPNEC. Several retrospective studies have reported the results of the utilization of temozolomide (TMZ) as a drug for the second-line treatment for EPNEC.

Methods:Patients with unresectable EPNEC that were resistant to platinum-based combination chemotherapy were recruited for a prospective phase II study of TMZ monotherapy. A $200 \mathrm{mg} / \mathrm{m}^{2}$ dose of $T M Z$ was administered from day 1 to day 5 , every four weeks. Response rate (RR) was evaluated as the primary endpoint. The presence of $0^{6}$-methylguanine DNA methyltransferase (MGMT) in EPNEC patients was also evaluated as exploratory research.

Results: Thirteen patients were enrolled in this study. Primary lesions were pancreas $(n=3)$, stomach $(n=3)$, duodenum $(n=1)$, colon $(n=1)$, gallbladder $(n=1)$, liver $(n=1)$, uterus $(n=1)$, bladder $(n=1)$, and primary unknown $(n=1)$. Each case was defined as pathological poorly differentiated neuroendocrine carcinoma from surgical resected and/or biopsied specimens. The median Ki67 labeling index was $60 \%$ (range: $22 \%-90 \%$ ). The RR was $15.4 \%$, progression free survival was 1.8 months (95\% confidence interval [CI], 1.0-2.7), overall survival (OS) was 7.8 months ( $95 \% \mathrm{Cl}, 6.0-9.5)$, and OS from first-line treatment was 19.2 months $(95 \% \mathrm{Cl}, 15.1-23.3)$. No grade 3 or 4 hematological toxicity had occurred and grade 3 nausea and appetite loss had occurred one case. One case presented MGMT deficiency and this case showed partial response.

Conclusion: TMZ monotherapy is a feasible, modestly effective, and safety treatment for patients with unresectable EPNEC following platinum-based chemotherapy. MGMT deficiency may be a reliable biomarker for the response of unresectable EPNEC to TMZ.

Trial registration: Registered at April 20, 2013. Registry number: UMIN000010549.

\section{Background}

Extrapulmonary poorly differentiated neuroendocrine carcinoma (EPNEC) originates in the gastrointestinal tract, gynecological, and urological organs as either a small-cell lung carcinoma (SCLC) or a large-cell neuroendocrine carcinoma (LCNEC), which are both similar to their respective well-known pulmonary counterparts (1). EPNEC is a rare disease; its incidence accounts for $10-20 \%$ of malignant neuroendocrine neoplasms (NENs) $(2,3)$. EPNEC has aggressive histological features that contribute to its poor prognosis and lethality (4). According to an analysis of 14,732 EPNEC cases from the Surveillance Epidemiology and End Results database, distant metastasis was diagnosed in $69 \%$ of patients with NEC, and the five-year survival rate for patients with distant metastasis was only $5.7 \%$ (5).

Systemic chemotherapy is the main treatment option for advanced EPNEC. Some clinical guidelines for treating EPNEC recommend platinum-based chemotherapy as the first-line treatment $(6,7)$. Response rate 
is about 30 to $50 \%$ however, progression free survival (PFS) of them is only 4 or 5 months $(8,9)$. Efficacy of platinum-based chemotherapy for EPNEC was limited, and second-line chemotherapy was necessary in one-half of all cases.

Second-line chemotherapy results in an RR of $11 \%$ and a PFS of only 2.1 months, retrospectively (9). Oxaliplatin-based chemotherapy, irinotecan-based chemotherapy and Amrubicin monotherapy is retrospectively performed, however the response rate is $18.8-38.5 \%$ and the PFS is $1.9-3.6$ months (10, $11,12,13,14)$.

Temozolomide (TMZ)-based chemotherapy is the most frequently used as a second-line regimen for unresectable NEC (8). The activity of TMZ in patients with unresectable NEC has been evaluated in several trials, however, most of them were retrospective studies or study target was not limited to NEC ( 15 , 16). Then, activity of TMZ for advanced NEC is still unclear. Moreover, there is no evidence of efficacy of TMZ for Japanese patients with advanced NEC because TMZ is not yet approved in Japanese health insurance system to utilize for advanced NEC. Prospective clinical study of TMZ monotherapy targeted at Japanese patients with advance NEC is worthy challenge.

The aim of this study was to determine the efficacy and safety of TMZ monotherapy for patients with unresectable EPNEC resistant to platinum-based chemotherapy. Evaluation of expression of 06methylguanine DNA methyltransferase (MGMT) considered as a chemo-sensitivity marker of TMZ was also conducted as an additional study.

\section{Methods}

\section{Study Design}

A phase II study using TMZ monotherapy as a second-line treatment in patients with unresectable EPNEC resistant to platinum-based chemotherapy, was conducted (from April 2013 to March 2017) (UMIN000010549) (IRB B130307033).

The prevalence of MGMT deficiency in EPNEC was evaluated, and a correlation was found between MGMT deficiency and treatment response to TMZ by immunohistochemistry as exploratory research. This trial was supported by Yokohama City University Hospital.

\section{Patient Selection}

Patients with pathologically confirmed, metastatic or recurrent poorly differentiated neuroendocrine carcinoma (WHO $2010 \mathrm{Ki} 67 \mathrm{LI}>20 \%$ Grade 3), who were previously treated with platinum-based first-line chemotherapy were eligible for this study if they met the following inclusion criteria: Eastern Cooperative Oncology Group PS of 0 or 1; age between 20-75 years; NEC with at least one measurable lesion based on Response Evaluation Criteria in Solid Tumors (RECIST); and adequate hematological, liver, and renal function (hemoglobin $>9.0 \mathrm{~g} / \mathrm{dl}$, white blood cell count $<10000 / \mathrm{mm}^{3}$, neutrophil count $>1500 / \mathrm{mm}^{3}$, 
platelet count $>100000 / \mathrm{mm}^{3}$, total bilirubin $<1.5$-fold the upper normal limit, serum transaminase $<$ threefold the upper normal limit, creatinine $<1.5$-fold the upper normal limit). The patients provided written informed consent. We excluded SCLC, LCNEC, mixed adenocarcinoma, neuroendocrine neoplasm (MiNEN), and adenocarcinoma with neuroendocrine features.

This was an open-label, single-center, non-randomized phase II study. All laboratory tests required to assess eligibility were completed within 7 days prior to the start of treatment. The treatment schedule involved the administration of temozolomide $\left(200 \mathrm{mg} / \mathrm{m}^{2}\right)$ on days $1-5$ every four weeks. The RR was evaluated as the primary endpoint and the PFS, overall survival (OS), and adverse events (AE) were evaluated as the secondary endpoints. AE were defined using the Common Terminology Criteria for Adverse Events version 4.0.

Immunohistochemistry

Immunohistochemistry was conducted on the formalin-fixed, paraffin-embedded (FFPE) tissue sections. We reacted $4-\mu \mathrm{m}$-thick sections of representative blocks with monoclonal antibodies against MGMT (MT 3.1; 1:25, GeneTex, Irvine, US). Nuclear MGMT expression was scored as either intact or deficient in tumor cells (17). Tumors were scored as intact when nuclear staining for MGMT was observed in any of the tumor cells. Tumors were scored as deficient when nuclear staining for MGMT was not observed in all tumor cells. Non-neoplastic cells served as an internal positive control in all tissue sections.

\section{Statistical Design}

SWOG's standard design (attained design) was used to determine the number of patients enrolled in the study. The null hypothesis stated that the overall RR would be $<5 \%$ and the alternative hypothesis stated that the overall RR would be $>30 \%$, the a error was determined to be $5 \%$ (one-tailed) and the $\beta$ error was determined to be $20 \%$ (one-tailed). The alternative hypothesis was established based on preferable data from previous reports. The sample size was set at 13 cases. The median survival time and corresponding $95 \%$ confidence intervals (Cls) for OS and PFS were estimated using the Kaplan-Meier method. PFS was defined as the time from day 1 of cycle 1 until the first event (progressive disease or death by any cause). If no such event occurred, data for that patient were censored on the day of the last imaging procedure. OS was defined as the time from day 1 of cycle 1 until death by any cause. If death did not occur, data were censored on the last day of survival confirmation. All analyses were performed using SPSS version 21.0 (IBM, New York, US).

\section{Results}

\section{Patients and Characteristics}

Between April 2013 and March 2017, 13 patients were enrolled in this study. Table 1 shows the patient characteristics at baseline. The median age of the patients was $65(40-75)$ years. Six patients were male and seven patients were female. Three cases had a performance status of 1 , and ten cases had a 
performance status of 0 . All cases showed metastatic and unresectable EPNEC. Four cases had undergone surgical resection of the tumor prior to treatment. The first-line chemotherapy regimens were classified as IP (irinotecan + Cisplatin) $n=8$, PE (Cisplatin + Etoposide) $n=4$, and Carboplatin + Etoposide $n=1$. Primary lesions were classified as pancreas $(n=3)$, stomach $(n=3)$, duodenum $(n=1)$, colon $(n=1)$, gallbladder $(n=1)$, liver $(n=1)$, uterus $(n=1)$, bladder $(n=1)$, and primary unknown $(n=1)$. The median Ki67 labelling index was 60\% (range 22-90\%).

\section{Efficacy}

The TMZ dose of $200 \mathrm{mg} / \mathrm{m}^{2}$ was administered on days 1-5 every four weeks. A total of 28 cycles of TMZ were administered to all patients, and the median number of cycles per patient was two (range, 1-4 RR was $15.4 \%$ and disease control rate was $23.1 \%(C R=0, P R=2, S D=1, P D=10)$ (Table 2). PFS was 1.8 months $(95 \% \mathrm{Cl}, 1.0-2.7)$ and OS was 7.8 months $(95 \% \mathrm{Cl}, 6.0-9.5)$. And OS from first-line treatment was 19.2 months ( $95 \% \mathrm{Cl}, 15.1-23.3)$. (Fig. 1,2).

Toxicity

The most common adverse events were hematological toxicities. There were no occurrences of grade 3 or 4 severe neutropenia, anemia, or thrombocytopenia. All hematological toxicities were non-severe (grade $1,2)$ toxicities; anemia occurred in 12 patients $(92 \%)$, thrombocytopenia occurred in 5 patients $(39 \%)$, and leucopenia occurred in one patient (8\%) (Table 3).

Of all severe non-hematological toxicities, only severe appetite loss and nausea were observed in one patient (8\%) (Table 3). Grade 1 and 2 non-hematological toxicities that occurred included: liver dysfunction in 3 patients (23\%), renal dysfunction in 3 patients (23\%), nausea in 5 patients (38\%), vomiting in 3 patients (23\%), diarrhea in 1 patient $(8 \%)$, general fatigue in 4 patients $(31 \%)$ and appetite loss in 6 patients $(46 \%)$.

Immunohistochemical analysis

FFPE tissues sections were obtained from all cases. Sufficient tissues for immunohistochemical analysis could not be obtained from two cases. As a result, eleven tissue samples were analyzed in total (Table 2). Ten cases were found to be intact and one case was found to be deficient (Fig. 3a, b). The deficient case had a primary liver NEC with multiple liver metastases and temporarily achieved a partial response (Fig. 3c, d).

\section{Discussion}

The activity of TMZ in patients with unresectable NEN has been evaluated in several trials, which showed interesting activity in terms of RR values, ranging from 14 to $70 \%(18,19,20)$. However, these clinical data describe TMZ-based chemotherapy for mainly well-to-moderately differentiated neuroendocrine tumor (NET) (WHO 2017: Grade1 and/or Grade2). Primary pancreatic NET shows a good response (43-70\%) to 
TMZ therapy with a long rate of progression-free survival (12-18 months) $(19,20)$. Recently, in a prospective randomized phase II study for pancreatic NET (G1 or G2) with TMZ monotherapy versus capecitabine and TMZ combination chemotherapy, the response rate was $33.3 \%$ vs $27.8 \%$ and the PFS was 22.7 months vs 14.4 months. TMZ and capecitabine combination chemotherapy shows a significantly better PFS than TMZ monotherapy (21). ENETS consensus guideline described TMZ-based chemotherapy should be considered for well-differentiated NEN included NET G3 (6).

However, TMZ-based chemotherapy for NEC is not sufficiently effective as an urgent treatment. In a previous study, TMZ monotherapy or TMZ in combination with capecitabine and bevacizumab for NEC showed a response rate of $0-57 \%$ and a PFS of $2.4-8.4$ months $(15,16,22)$. In general, second- or thirdline chemotherapy treatment for EPNEC has a response rate of $0-18.8 \%$ and a PFS of $2.1-3.8$ months (9, $14,16,23)$. In this study, we estimated the response rate as $15.4 \%$ and the PFS as 1.8 months. Here, TMZ monotherapy was undertaken in six cases as a second-line treatment, but in the other seven cases it was undertaken as the third- or salvage- line treatment. Patients in this study undergoing TMZ monotherapy showed a more advanced disease than the patients from the remainder of the TMZ studies reported so far.

TMZ monotherapy is a mild toxic drug, and incidents of severe toxic events have been rare in previously reported studies. In this study, there were no grade 3 or 4 hematological and few non-hematological toxicities. Patients received some chemotherapy for a long duration (about six months; detailed data not shown) and their general condition was no better than that of those in previously reported studies. No severe toxicity appeared for these advanced patients. Recently, many studies for unresectable NEN were performed using TMZ and capecitabine combination therapy that showed the same results and confirmed the mild toxicity of this therapy.

MGMT deficiency was confirmed by immunohistochemistry analysis and a case of deficient was response to TMZ monotherapy in this study. MGMT expression in the tumor cells was assessed as a predictive biomarker for alkylating agents. One mechanism of resistance to alkylating agents is an increase in the expression of the DNA repair enzyme MGMT. If expression of MGMT is decreased by methylation of the MGMT promotor region, DNA repair is decreased. A decrease in MGMT expression, which frequently occurs during carcinogenesis, may increase the sensitivity of tumor cells to alkylating agents that induce DNA damage, thus increasing the response to alkylating agents. Many previous studies have described the relationship between MGMT expression and response of NENs $(17,24)$. In our study, the analysis of the MGMT deficient case showed shrinkage of multiple liver tumors after two treatment cycles. However, the tumors rapidly regrew after two cycles. According to another recent study, metronomic TMZ monotherapy (one-week-on/one-week-off treatment) for pancreatic NEC with MGMT deficiency is a good option for second-line chemotherapy (25). We consider TMZ monotherapy is a potential treatment option for NEC with MGMT deficiency as a second- or third-line treatment.

This study has some limitations. First, the sample size was small and primary lesions were heterogeneous. Most prior studies were designed for unresectable pancreatic NEN or GEP-NEN. EPNEC 
shows a high degree of heterogeneity, so the efficacy of TMZ may differ according to primary lesions. Second, we evaluated MGMT status with IHC. In a clinical setting, MGMT status can be evaluated by assessing MGMT protein expression using immunohistochemistry $(\mathrm{IHC})$ or by determining MGMT promotor methylation using a methylation assay. Some past reports suggest that concordance is relatively low between IHC and MSP assessments of MGMT status (26).

\section{Conclusion}

Our study revealed that salvage-line TMZ monotherapy was a safe and marginally effective treatment for patients with unresectable EPNEC after the failure of first-line platinum-based chemotherapy. A more effective second-line treatment option is necessary for EPNEC, but chemotherapy with TMZ combined with another agent may serve as a feasible and effective treatment option for the treatment of EPNEC, especially in the presence of MGMT deficiency.

\section{Abbreviations}

EPNEC: Extrapulumonary neuroendocrine carcinoma; TMZ: Temozolomide; MGMT: $0^{6}$-methylguanine DNA methyltransferase; RR: Response rate; PFS: Progression free survival; OS: Overall survival; NENs: Neuroendocrine neoplasms

\section{Declarations}

Ethics approval and consent to participate

This study was conducted in accordance with the principles expressed in the Declaration of Helsinki. The main protocol of this study was approved by the institutional review board of Yokohama City University (Trial No. B130307033 and B160101021) at March $8^{\text {th }} 2013$ and February $5^{\text {th }} 2016$. Protocol amendments have been made in the study period. The latest version (version 3.0) of study protocol have been reviewed and approved by the institutional review board in Yokohama City University. Written informed consent will be obtained from all the participants.

Consent for publication

Not applicable.

Availability of data and materials

The datasets used and/or analyzed during the current study are available from the corresponding author on reasonable request.

Competing interests

The authors declare that they have no competing interests. 
Funding

No funding was received.

Authors' contributions

NK drafting of the article

YT, NO, AS, MT, YH: conception and design, final approval of critical revision for important intellectual content

YI: final approval of the article

All authors have read and approved the manuscript.

Acknowledgements

We thank the patients and their families who participated in this study. We would like to thank Editage (www.editage.jp) for English language editing. This trial was supported by Yokohama City University

\section{References}

1) Volante M, Birocco N, Gatti G, et al. Extrapulmonary neuroendocrine small and large cell carcinomas: a review of controversial diagnostic and therapeutic issues. Hum Pathol. 2014;45:665-73.

2) Lepage $C$, Ciccolallo $L$, De-Angelis $R$, et al. European disparities in malignant digestive endocrine tumors survival. Int J Cancer. 2010;126:2928-2934.

3) Kang $\mathrm{H}$, OConnell JB, Leonardi MJ. Rare tumors of the colon and rectum: a national review. Int J Colorectal Dis. 2007;22:183-189.

4) Sorbye H, Strosberg J, Baudin E,et al. Gastroenteropancreatic high-grade neuroendocrine carcinoma. Cancer. 2014;120:2814-23.

5) Dasari A, Mehta K, Byers LA,et al. et al. Comparative study of lung and extrapulmonary poorly differentiated neuroendocrine carcinomas: A SEER database analysis of 162,983 cases. Cancer 2018;124:807-815.

6) Pavel M, O'Toole D, Costa F, et al. ENETS consensus guidelines update for the management of distant metastatic disease of intestinal, pancreatic, bronchial neuroendocrine neoplasms (NEN) and NEN of unknown primary site. Neuroendocrinology 2016;103:172-185

7)Kulke MH, Shah MH, Benson AB $3^{\text {rd }}$, et al. Neuroendocrine tumors, version $1.2015 \mathrm{~J}$ Natl Compr Canc Netw. 2015;13:78-108. 
8) Sorbye H, Welin S, Langer S.W, et al. Predictive and prognostic factors for treatment and survival in 305 patients with advanced gastrointestinal neuroendocrine carcinoma

(WHO G3): The NORDIC NEC study. Ann Oncol. 2013;24:152-160.

9) Yamaguchi T, Machida N, Morizane C, et al.

Multicenter retrospective analysis of systemic chemotherapy foradvanced neuroendocrine carcinoma of the digestive system. Cancer Science. 2014;105:1176-1181.

10) Bajetta E, Catena L, Procopio G,et al. Are capecitabine and oxaliplatin (XELOX) suitable treatments for progressing low-grade and high-grade neuroendocrine tumours? Cancer Chemother Pharmacol. 2007;59:637-42.

11) Hentic $O$, Hammel $P$, Couvelard A,et al. FOLFIRI regimen: an effective second-line chemotherapy after failure of etoposide-platinum combination in patients with neuroendocrine carcinomas grade 3 . Endocr. Relat. Cancer. 2012;19:751-757.

12) Nio $K$, Arita $S$, Isobe $T$,et al. Amrubicin monotherapy for patients with extrapulmonary neuroendocrine carcinoma after platinum-based chemotherapy. Cancer Chemother Pharmacol. 2015 ;75:829-35.

13) Ando $\mathrm{T}$, Hosokawa A, Yoshita $\mathrm{H}$, et al. Amrubicin Monotherapy for Patients with Platinum-Refractory Gastroenteropancreatic Neuroendocrine Carcinoma. Gastroenterol Res Pract. 2015;2015:425876.

14) Araki $T$, Takashima $A$, Hamaguchi $T$, et al. Amrubicin in patients with platinum-refractory metastatic neuroendocrine carcinoma and mixed adenoneuroendocrine carcinoma of the gastrointestinal tract. Anticancer Drugs. 2016;27:794-9.

15) Welin S, Sorbye H, Sebjornsen S, et al. Clinical effect of temozolomide-based chemotherapy in poorly differentiated endocrine carcinoma after progression on first-line chemotherapy. Cancer. 2011;117:461722.

16) Olsen IH, Sorensen JB, Federspiel B, et al. Temozolomide as second or third line treatment of patients with neuroendocrine carcinoma. Scientific World Journal 2012; 2012: 170496.

17) Kulke MH, Hornick JL, Frauenhoffer $C$, et al. O6-methylguanine DNA methyltransferase deficiency and response to temozolomide-based therapy in patients with neuroendocrine tumors. Clin Cancer Res 15: $338-345 ; 2009$

18) Ekeblad S, Sunddia A, Janson ET, et al. Temozolomide as monotherapy is effective in treatment of advanced malignant neuroendocrine tumors. Clin Cancer Res. 2007;13:2986-75.

19) Strosberg JR, Fine RL, Choi J, et al. First-line chemotherapy with capecitabine and temozolomide in patuents with metastatic pancreatic endocrine carcinoma. Cancer. 2011;117:268-75. 
20) Saif MW, Kaley K, Brennan M, et al. A retrospective study of capecitabine/temozolomide (CAPTEM) regimen in the treatment of metastatic pancreatic neuroendocrine tumors (pNETs) after failing previous therapy. JOP. 2013;14:498-501.

21) Kunz PL, Catalano PJ, Nimeiri $\mathrm{H}$, et al. A randomized study of temozolomide or temozolomide and capecitabine in patients with advanced pancreatic neuroendocrine tumors: A trial of the ECOG-ACRIN Cancer Research Group (E2211). J Clin Oncol. 2018; 36, (suppl; abstr 4004)

22) Owen $\mathrm{DH}$, Alexander $\mathrm{AJ}$, Konda $\mathrm{B}$, et al. Combination therapy with capecitabine and temozolomide in patients with low and high grade neuroendocrine tumors, with an exploratory analysis of $\mathrm{O}_{6}$ methylguanine DNA methyltransferase as a biomarker for response. Oncotarget. 2017;8:104046-104056.

23) Apostolidis L, Bergmann F, Jäger D, et al. Efficacy of topotecan in pretreated metastatic poorly differentiated extrapulmonary neuroendocrine carcinoma. Cancer Medicine. 2016; 5:2261-2267.

24) Campana D, Walter T, Pusceddu S, et al. Correlation between MGMT promoter methylation and response to temozolomide-based therapy in neuroendocrine neoplasms: an observational retrospective multicenter study. Endocrine. 2018;60:490-498.

25) De Divitiis C, von Arx C, Grimaldi AM, et al. Metronomic temozolomide as second line treatment for metastatic poorly differentiated pancreatic neuroendocrine carcinoma. J Transl Med. 2016;14:113.

26) Cives $M$, Ghayouri M, Morse B, et al. Analysis of potential response predictors to capecitabine/temozolomide in metastatic pancreatic neuroendocrine tumors. Endocr Relat Cancer. 2016;23:759-67.

\section{Tables}

Due to technical limitations the Tables are available as a download in the Supplementary Files.

\section{Figures}




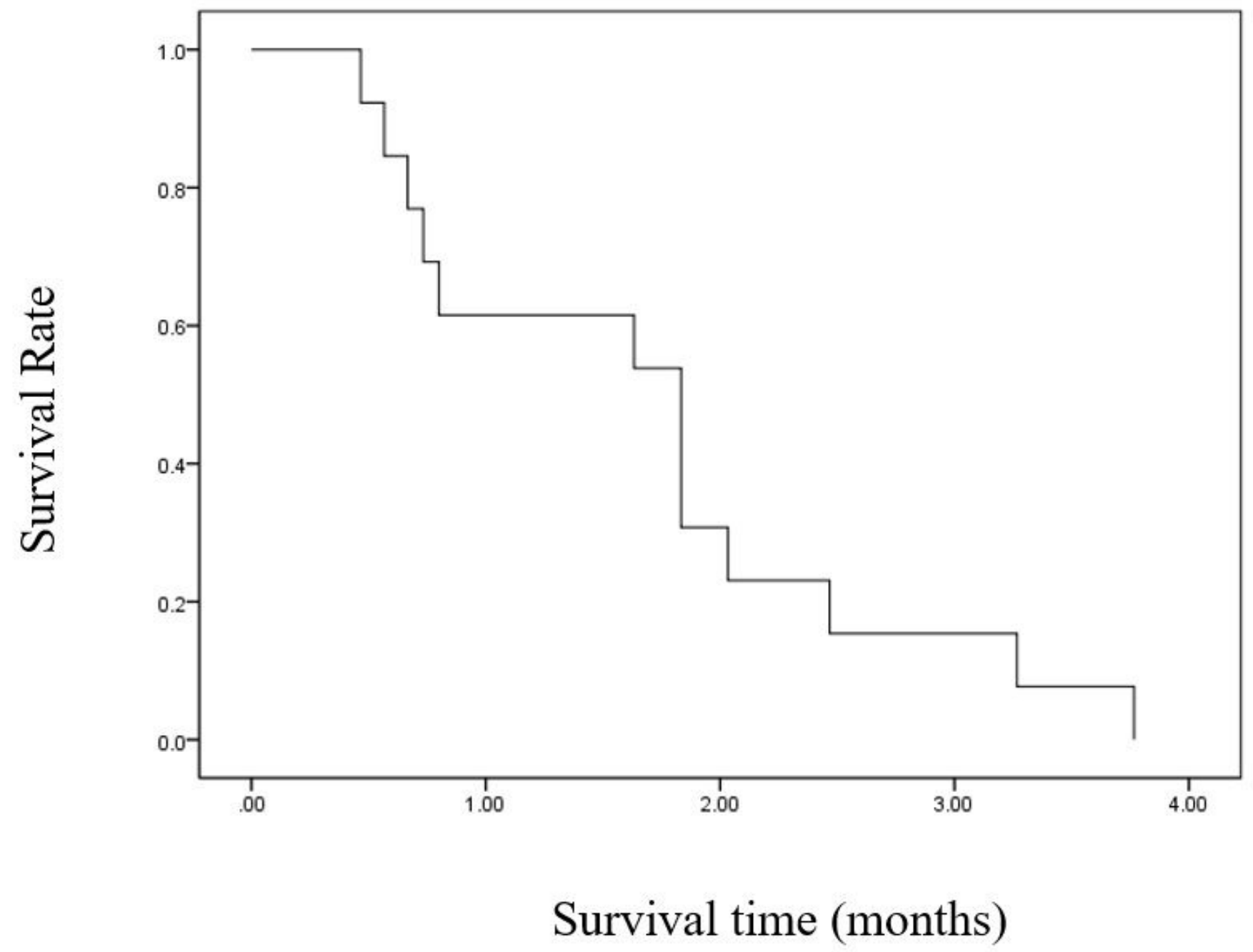

Figure 1

Kaplan-Meier curve for progression free survival. The median progression-free survival was 1.8 months (95\% confidence interval. 1.0-2.7). No patient data was censored. 


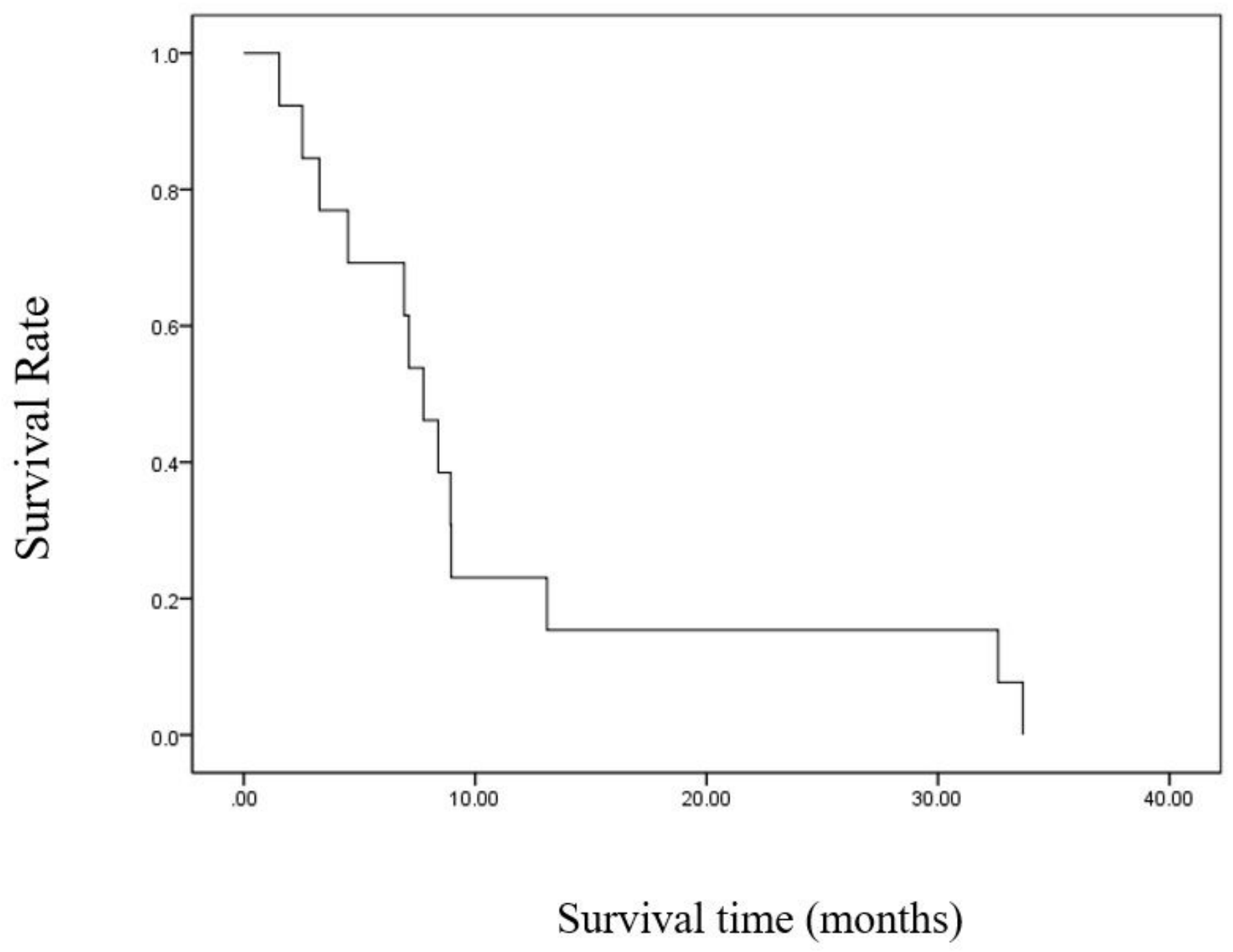

Figure 2

Kaplan-Meier curve for overall survival. The median overall survival was 7.8 months ( $95 \%$ confidence interval. 6.0-9.5). No patient data was censored. Figure 3a. This serial section was primary lesion of gastric neuroendocrine carcinoma with multiple liver metastatic case (Case 3). Immunohistochemical findings revealed tumor cells were diffuse and moderately stained by MGMT protein. This case was defined intact. 

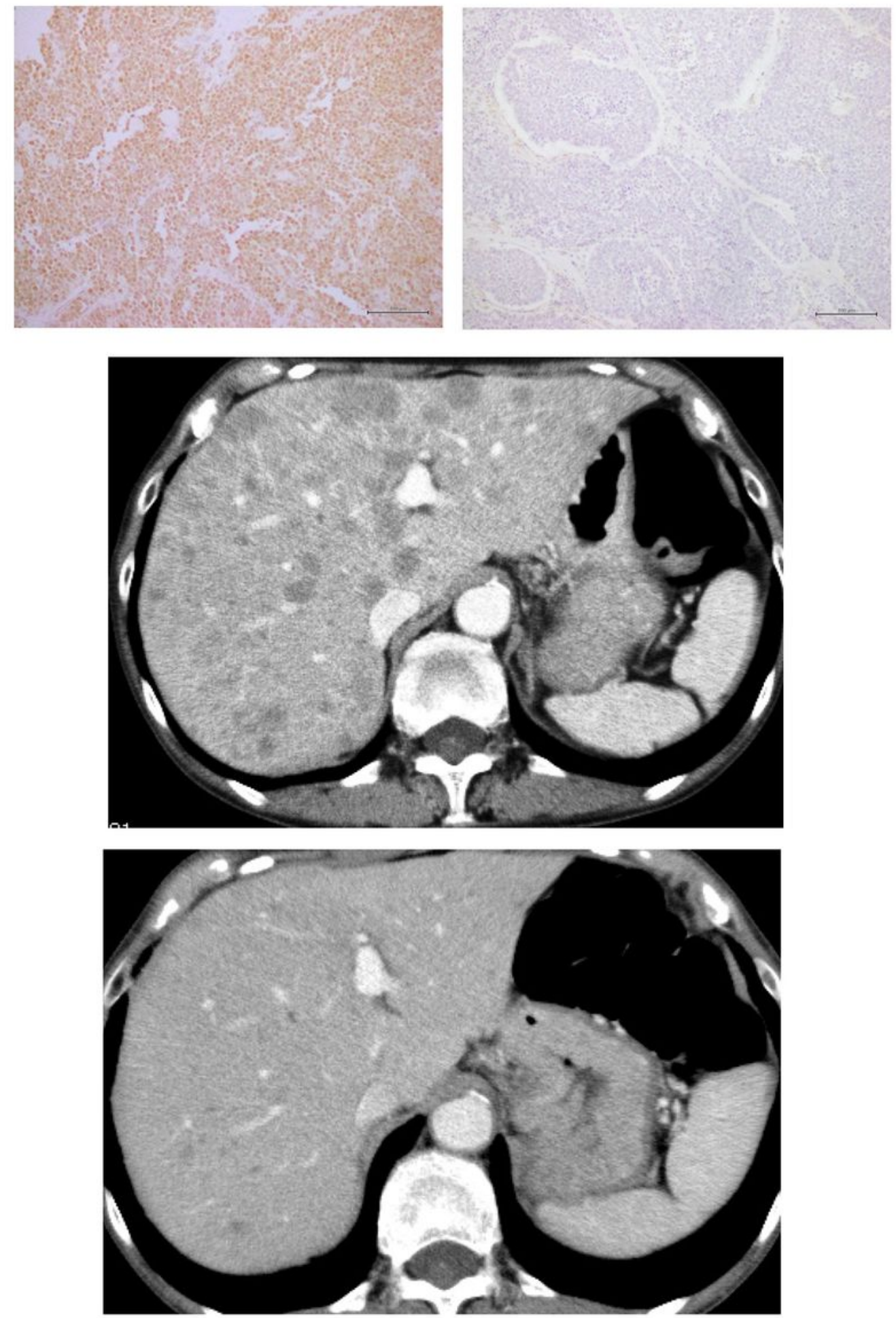

\section{Figure 3}

a. This serial section was primary lesion of gastric neuroendocrine carcinoma with multiple liver metastatic case (Case 3). Immunohistochemical findings revealed tumor cells were diffuse and moderately stained by MGMT protein. This case was defined intact. b. This serial section was liver lesion of hepatic neuroendocrine carcinoma case (Case 9). Immunohistochemical findings revealed tumor cells were not stained by MGMT protein diffusely. This case was defined deficiency. c. CT findings revealed 
multiple liver metastasis before the treatment with temozolomide (Case 9). d. CT findings revealed multiple liver metastasis after two cycle treatments with temozolomide. Multiple liver tumors were remarkable shrinkage (Case 9).

\section{Supplementary Files}

This is a list of supplementary files associated with this preprint. Click to download.

- Tablenew.pptx 